\title{
Atraumatic brachial plexopathy following intravenous heroin use
}

\author{
P. A. EVANS \& H. T. MILLINGTON \\ Accident and Emergency Department, Charing Cross Hospital Fulham Palace Road, \\ London W6 8RF
}

\section{SUMMARY}

A 32-year-old man presented to the accident \& emergency (A\&E) department complaining of an inability to use his left arm and shoulder. The previous day he had injected heroin intravenously into his left antecubital vein. Examination revealed signs of a left-sided brachial plexus lesion. There was no history or sign of trauma. Neurological investigation revealed motor and sensory loss compatible with a complete brachial plexus lesion. He exhibited a very rare condition, asymmetrical atraumatic brachial plexopathy, thought to result from an inflammatory cause, which not only affects the brachial, but also other plexi or individual nerves in the body and thought to be related to repeated intravenous use of heroin. This is a condition for which there is no specific treatment but which usually resolves spontaneously in the absence of continuing heroin misuse.

\section{INTRODUCTION}

A 32-year-old right handed bricklayer presented to the A\&E department with paralysis and loss of sensation in his left arm and shoulder. The previous day he had given himself an intravenous injection of heroin. He had woken that morning with a complete paralysis of his left arm. He was a known heroin user with a history of regular previous use.

He had managed to abstain from using heroin for 8 months, but 1 week previously he had recommenced intravenous injection of heroin. His friends had also used heroin from the same source that day and previously and had suffered no ill effects following it. He was otherwise fit and well and had no headache or any other neurological symptoms. There, was no history of trauma or any other features in the history to suggest the aetiology of this paralysis. In addition to the 
paralysis, he had also noticed loss of sensation in the arm and, more recently, dista pain and tingling, particularly if he pressed immediately above the left clavicle $\frac{3}{0}$ General examination was unremarkable. The cranial nerves were normal. In the limbs, the abnormalities were confined to the left arm and shoulder. The left arm was flaccid with no movement at all below the left shoulder. There was somed strength in the supraspinatus, reasonable strength in infraspinatus and rhomboid but no strength at all in the deltoid, pectoral, serratus anterior or latissimus dors? muscles. There were no reflexes in the left arm.

Sensory loss was profound with a virtually complete anaesthesia in a distribution compatible with a complete brachial plexus lesion, except for some sparing ove $\vec{P}$ the deltoid in the $\mathrm{C} 5$ distribution. Examination of his cardiovascular, respiratory $\vec{\omega}$ and gastrointestinal systems were unremarkable. Investigations of FBC., ESR electrolytes, blood sugar, ECG, chest and cervical spine X-rays were all normal. $\mathrm{He}$. was referred to the neurologists who performed electromyographic studies which showed widespread neurogenic changes extending from $\mathrm{C} 5$ to $\mathrm{T} 1$, with sensorje and motor changes, consistent with a brachial plexus neuropathy. On examination by the neurologists a week later, there had been a slight improvement with some? fasciculation in his triceps muscle, but there was still quite marked muscle weak $=$ ness $3 / 5$, with very little sensory change. Physiotheraphy and occupational therap $x_{\infty}^{5}$ were arranged and an out-patient appointment made to assess progress. He faile $\frac{\mathbb{P}_{0}}{0}$ to keep the appointment and was lost to follow up.

\section{DISCUSSION}

A variety of neurological complications have been reported and documented concerning intravenous heroin users including both acute and non-acute poly흥 neuropathies. However, atraumatic plexopathies following intravenous heroin use due to an inflammatory process are extremely rare and have only been documented on a few occasions. A particularly noteworthy point regarding this patient con cerns the fact that, generally speaking, brachial plexopathies of this nature are symmetrical and of a gradual onset, whereas this patient's symptoms and signs were asymmetrical and were of a more immediate nature. The aetiology and: pathogenesis of this neurological complication is uncertain and an inflammatorye. process is thought by many neurologists to be the underlying cause.

Various authors (Richter et al., 1969, 1970; Antonini et al., 1989a, b) have showr? that plexopathies of this nature can be caused by contaminants and adulterants such as heavy metals, indeed lead poisoning as a cause of brachial plexopathy has been documented before. However, plexopathies due to heavy metals are usuall, symmetrical and are of gradual onset with other clinical sequelae and this did nop occur in this case. Other than the association with intravenous heroin use, no other cause was found to explain this man's neurological abnormalities. 


\section{REFERENCES}

Antonini G., Palmieri G., Spagnoli L. G. \& Millefiorini M. (1989a) Lead brachial neuropathy in heroin addiction. Clinical Neurology and Neurosurgery 9, 1-2.

Antonini G., Palmieri G., Millefiorini E., Spagnoli L. G. \& Millefiorini M. (1989b) Lead poisoning during heroin addiction. Italian. Journal of Neurological Science 10, 105-108.

Richter R. W. \& Baden M. N. (1969) Neurological complication of heroin addiction. Transactions of the American Neurological Society 94, 330-332.

Richter R. W., Baden M. N. \& Pearson, J. (1970) Clinical and neuropathological correlates of heroin addiction. Journal of the American Medical Association Medical News 212, 967. 